\title{
A Sparse Matrix Approach for Simultaneous Quantification of Nystagmus and Saccade
}

\author{
Sunil L. Kukreja, Leland S. Stone and Richard D. Boyle
}

\begin{abstract}
The vestibulo-ocular reflex (VOR) consists of two intermingled non-linear subsystems; namely, nystagmus and saccade. Typically, nystagmus is analysed using a single sufficiently long signal or a concatenation of them. Saccade information is not analysed and discarded due to insufficient data length to provide consistent and minimum variance estimates.

This paper presents a novel sparse matrix approach to system identification of the VOR. It allows for the simultaneous estimation of both nystagmus and saccade signals. We show via simulation of the VOR that our technique provides consistent and unbiased estimates in the presence of output additive noise.
\end{abstract}

\section{INTRODUCTION}

A bifurcating or switched system is one that switches between various modes of operation via internal or external influences. When a switch occurs from one subsystem to another, a discontinuity may result followed by a smooth progression under the new state [1].

The vestibulo-ocular reflex (VOR) is a well known example of a biological system that exhibits non-linear bifurcating behavior [2]. Although typically analysis of VOR dynamics is accomplished by relying on linear a priori modelling techniques, such models do not account for the rich dynamic behavior due to non-linearities [3]. When studying subjects known to have vestibular deficit such models are of limited use due to mode interactions through initial conditions.

Recently, Kukreja et al. [4] showed the VOR can be accurately modelled by the NARMAX (Non-linear AutoRegressive, Moving Average eXogenous) structure. NARMAX models describe the current system output in terms of past inputs, outputs and errors and may include a variety of nonlinear terms and, as such, they are suitable to describe the input-output relationship of non-linear switched systems [5].

Moreover, Kukreja et al.[4] developed a modified extended least-squares (MELS) algorithm to estimate unbiased parameter values of non-linear Hammerstein structure multimode systems. This approach uses multiple short data segments of both nystagmus and saccade data, which requires the signal be classified into modes of operation [6]. The classified signal is concatenated, with appropriate modelling of bifurcation points, providing parameter estimates of each category of eye movement. This two-step process adds overhead to data analysis, which can be significant for sufficiently

S.L. Kukreja is with the Research Engineering Directorate, NASA Dryden Flight Research Center, Edwards, CA, USA Sunil.L.Kukreja@nasa.gov

L.S. Stone is with the Human Systems Integration Division, NASA Ames Research Center, Moffett Field, CA, USA Leland. S. Stone@nasa.gov

R.D. Boyle is with the Space Biosciences Division, NASA Ames Research Center, Moffett Field, CA, USA Ri chard. Boyle@nasa.gov long data records and batch processing. However, it can be reduced to one step by exploiting the nature and efficiency of sparse matrices.

A sparse matrix or signal is characterised as one whose values are dominated by zeros and a few nonzero elements. A sparse structure offers the advantage of being easily compressed. By only storing non-zero elements a significant reduction in memory allocation can be realised [7]. This feature allows the implementation of special computational techniques to take advantage of the large number of zeros to speed up computations [7], [8]. In fact, some large sparse matrices do not lend themselves to efficient manipulation by standard algorithms designed for dense structures [9], [10]. In this paper, we show the utilisation of a sparse matrix approach is ideally suited for system identification of switched systems with application to the VOR.

Understanding the VOR is important for NASA's Human Research Program to address (i) Risk of Impaired Control of Spacecraft Associated Systems and (ii) Immediate Vehicle Egress Due to Vestibular/Sensorimotor Alterations Associated with Space Flight [11]. The ability to objectively quantify mechanisms that control VOR will support NASAs human exploration missions by providing methods to predict spatial disorientation and develop individualised countermeasures for astronaut crews that must perform critical space operations under varied gravito-inertial conditions such as launch, landing and orbital maneuvering [12].

The organisation of this paper is as follows. Section II introduces the NARMAX model structure. Section III introduces the VOR system, which is well known to exhibit bifurcating behaviour. A non-sparse matrix approach, namely, the MELS algorithm is summarised in §IV whilst a sparse matrix approach is developed in $\S \mathrm{V}$. This sparse matrix formulation is capable of simultaneously quantifing both nystagmus and saccade data. Section VI provides results of the proposed algorithm on a simulated VOR model. Section VII summarizes the conclusions of our study.

\section{NARMAX STRUCTURE}

A NARMAX structure's input-output relationship is modelled as a non-linear difference equation of the form [13].

$$
\begin{array}{r}
y(n)=f^{l}\left[y(n-1), \ldots, y\left(n-n_{y}\right), u(n), \ldots,\right. \\
\left.u\left(n-n_{u}\right), e(n-1), \ldots, e\left(n-n_{e}\right)\right]+e(n)
\end{array}
$$

where $f^{l}$ is a non-linear mapping, $u$ is the exogenous input, $y$ is the output, and $e$ is the innovation, or uncontrolled input.

The derivation of the NARMAX model (Eqn.1) was based on the zero-initial-state response. However, there are cases 
where it may be required to extend it to the non-zeroinitial-state case [5]. This approach was taken to extend the NARMAX formulation to model bifurcating systems as [4]

$$
\begin{aligned}
& y_{m}(n)=f^{l}\left[y_{m}(n-1), \ldots, y_{m}\left(n-n_{y}\right), u_{m}(n), \ldots,\right. \\
& u_{m}\left(n-n_{u}\right),, \delta_{m}(n), \ldots, \delta_{m}\left(n-n_{\delta}\right), e_{m}(n-1), \ldots, \\
& \left.e_{m}\left(n-n_{e}\right)\right]+e_{m}(n) \text { for } m=1,2, \ldots, \mathscr{M} \quad \forall \text { finite } \mathscr{M}
\end{aligned}
$$

where $m$ represents the modes of operation, $u_{m}, y_{m}, e_{m}$ are as defined previously, and $\delta_{m}$ are Kronecker impulse functions applied at the beginning of each data segment. Notice the lagged impulse values account for non-zero-initial-states. This modified description of the NARMAX structure allows it to model non-linear bifurcating systems.

\section{MODEL OF VOR DYNAMICS}

VOR dynamics are a result of ocular responses to head perturbations. These responses are classified as slow or fast, according to their average speed characteristics. The VOR has a time record response with a saw wave pattern called ocular nystagmus (Fig. 1) [3], [14]. This saw wave pattern is

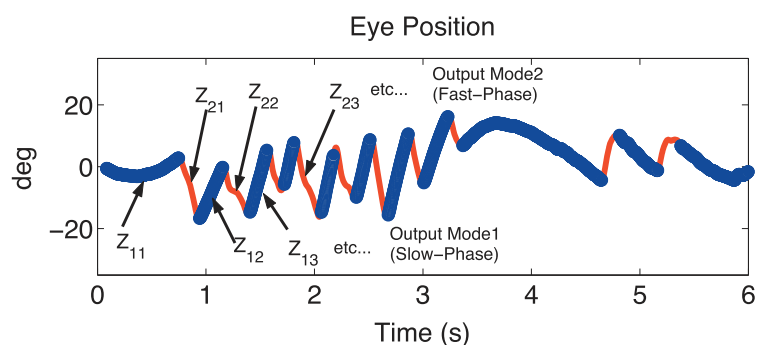

Fig. 1. VOR output. Blue Segments: slow-phase. Red Segments: fast-phase.

a result of the dual mode switching nature of the VOR. The saw wave pattern represents the slow-phase which stabilizes the eye in space and the fast-phase which re-orients the eye in the direction of head rotation [3], [15], [16]. Fig. 2 shows a typical output of the VOR illustrating its dual mode switching character.

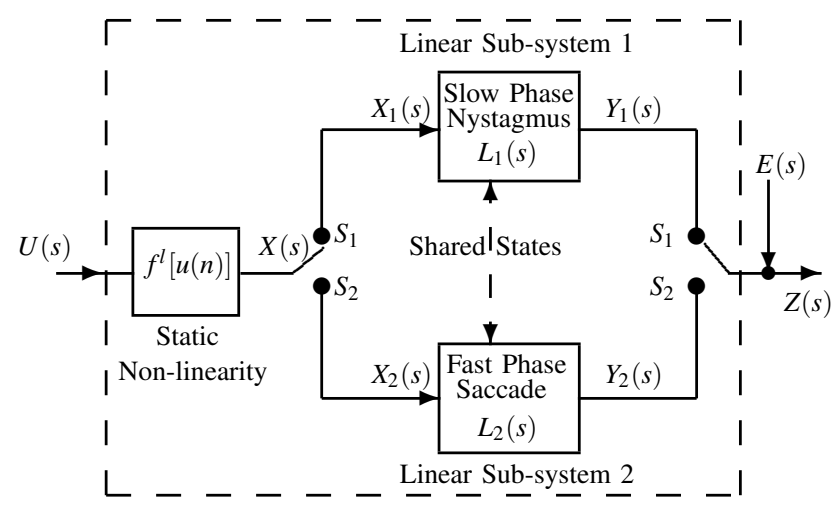

Fig. 2. Dual mode system describing vestibular dynamics. Switch position $S_{1}$ : slow-phase (nystagmus). Switch position $S_{2}$ : fast-phase (saccade)

\section{A. Continuous-Time Representation of VOR Model}

In Fig. 2 let $f^{l}(\cdot), Y_{1}(s)$ and $Y_{2}(s)$ are defined as [4]

$$
\begin{aligned}
f^{l}(\cdot) & =a+b u(n)+c u^{2}(n)+d u^{3}(n), \\
Y_{1}(s) & =\frac{K_{1}}{\tau_{1} s+1} X_{1}(s)+\frac{Y_{1 i}(o)}{\tau_{1} s+1} \\
& =\frac{G_{1}}{s+p_{1}} X_{1}(s)+\frac{Y_{1 i}(o) / \tau_{1}}{s+p_{1}} ; i=1, \cdots, q, \\
Y_{2}(s) & =\frac{K_{2}}{\tau_{2} s+1} X_{2}(s)+\frac{Y_{2 \ell}(o)}{\tau_{2} s+1} \\
& =\frac{G_{2}}{s+p_{2}} X_{2}(s)+\frac{Y_{2 \ell}(o) / \tau_{2}}{s+p_{2}} ; \ell=1, \cdots, r
\end{aligned}
$$

where $Y_{1}(s)$ and $Y_{2}(s)$ are first-order approximations for slow and fast-phase modes, $Y_{1 i}$ and $Y_{2 \ell}$ represent the initial conditions and $q, r$ are the number of switches.

B. Discrete-Time Representation of VOR Model

A NARMAX description of VOR slow-phases, $y_{1}(n)$, and fast-phases, $y_{2}(n)$ of the model in Eqn. 3 is [4]

$$
\begin{aligned}
y(n) & = \begin{cases}y_{1}(n) & \text { Dynamic Mode } S_{1} \\
y_{2}(n) & \text { Dynamic Mode } S_{2}\end{cases} \\
y_{1}(n) & =\beta_{1}+\beta_{2} y_{1}(n-1)+\beta_{3}[u(n)+u(n-1)] \\
& +\beta_{4}\left[u^{2}(n)+u^{2}(n-1)\right]+\beta_{5}\left[u^{3}(n)+u^{3}(n-1)\right] \\
& +\kappa_{1} \delta_{11}(n-j)+\cdots+\kappa_{i} \delta_{1 i}\left(n-j_{i}\right) ; i=1, \cdots, q \\
y_{2}(n) & =\vartheta_{1}+\vartheta_{2} y_{2}(n-1)+\vartheta_{3}[u(n)+u(n-1)] \\
& +\vartheta_{4}\left[u^{2}(n)+u^{2}(n-1)\right]+\vartheta_{5}\left[u^{3}(n)+u^{3}(n-1)\right] \\
& +\lambda_{1} \delta_{11}(n-k)+\cdots+\lambda_{\ell} \delta_{1 \ell}\left(n-k_{\ell}\right) ; \ell=1, \cdots, r .
\end{aligned}
$$

where $\delta$ is the Kronecker impulse function, $j, k$ are the lags on the $\delta_{1 i}$ th and $\delta_{2 \ell}$ th impulse and $q, r$ are the number of data segments of sub-system one and two, respectively.

Table I shows the relationship of these discrete-time (DT) parameters to the underlying continuous-time (CT) parameters in Eqn. 3.

\begin{tabular}{cc}
\hline DT Coefficient & Relationship to CT \\
\hline$\beta_{1}, \vartheta_{1}$ & $\frac{\left(2 G_{1,2}, 2 T\right)}{2+p_{1,2} T}$ \\
$\beta_{2}, \vartheta_{2}$ & $\frac{-\left(-2+p_{1,2} T\right)}{2+p_{1,2} T}$ \\
$\beta_{3}, \vartheta_{3}$ & $\frac{\left(G_{1,2} b T\right)}{2+1,2 T}$ \\
$\beta_{4}, \vartheta_{4}$ & $\frac{\left(G_{1,2} C T\right)}{2+1,2 T}$ \\
$\beta_{5}, \vartheta_{5}$ & $\frac{\left(G_{1,2} d T\right)}{2+\vartheta_{1,2} T}$ \\
$\kappa_{1}, \lambda_{1}$ & $\frac{\left(Y_{1,2}(0) p_{1,2}\right)}{2+p_{1,2} T}$ \\
\hline
\end{tabular}

TABLE I

DISCRETE-TIME RELATIONSHIP OF NARMAX MODEL PARAMETERS TO UNDERLYING CONTINUOUS-TIME PARAMETERS.

\section{NON-SPARSE MATRIX APPROACH}

The steady-state response of a system can be estimated using the extended least-squares (ELS) algorithm [17]. However, ELS cannot be applied to switched systems since it does not account for the transients which occur at each switch time and as a result will produce biased estimates.

The MELS algorithm corrects for bias by including columns in the regressor matrix to account for initial conditions as

$$
\Phi=\left[\Psi_{z u \hat{\varepsilon}} \mid \Psi_{\delta}\right]
$$


$\Phi$ is a partitioned regressor matrix where $\Psi_{z u \hat{\varepsilon}}$ is a function of input-output and prediction errors. The extension, $\Psi_{\delta}$, represents the effects of initial conditions when a switch occurs. This yields a least-squares formulation for nystagmus and saccade response as

$$
Z_{1}=\Phi_{1} \theta_{1} \quad \text { and } \quad Z_{2}=\Phi_{2} \theta_{2}
$$

where $Z_{1,2}$ is the measured output, $\theta_{1,2}$ the unknown system parameters. Notice the expression given in Eqn. 6 is a nonsparse matrix approach and, as such, both fast and slow phase need to be analysed separately.

\section{SPARSE MATRIX APPROACH}

To simplify parameter estimation and increase computational efficiency a sparse matrix approach can be used. The expressions shown in Eqns. 4-6 readily lend themselves to a sparse matrix formulation [18]. Simultaneous analysis of both nystagmus and saccade signals is accomplished by constructing a diagonal block-oriented data matrix and exploiting its natural sparseness as

$$
\left[\begin{array}{l}
Z_{1} \\
Z_{2}
\end{array}\right]=\left[\begin{array}{cc}
\Phi_{1} & 0 \\
0 & \Phi_{2}
\end{array}\right]\left[\begin{array}{l}
\theta_{1} \\
\theta_{2}
\end{array}\right]
$$

or more compactly as

$$
\tilde{Z}=\tilde{\Phi} \tilde{\theta}
$$

A solution to the extended parameter set in Eqn. 8 is

$$
\tilde{\theta}=\left(\tilde{\Phi}^{T} \tilde{\Phi}\right)^{-1} \tilde{\Phi}^{T} \tilde{Z}
$$

This novel analysis technique achieves efficient computation of slow and fast-phase signals concurrently.

\section{SIMULATIONS \& RESULTS}

The accuracy of the sparse matrix parameter estimation algorithm was validated by simulating the VOR model (Fig. 2 ) in continuous-time using Simulink. The parameters used in the simulation were typical values found in experiments and are shown in Table II [3].

\begin{tabular}{cc|cc}
\hline CT System Coeff. & Value & CT NL Coeff. & Value \\
\hline$\tau_{1}$ & $15.0 \mathrm{~s}$ & $a$ & $3.00 \times 10^{-1}$ \\
$\tau_{2}$ & $50.0 \mathrm{~ms}$ & $b$ & 1.20 \\
$K_{1}$ & -9.43 & $c$ & $-3.00 \times 10^{-3}$ \\
$K_{2}$ & 0.222 & $d$ & $-1.50 \times 10^{-5}$ \\
$\mathrm{~T}$ & $1.67 \times 10^{-3} \mathrm{~s}$ & & \\
\hline
\end{tabular}

TABLE II

LEFT: CONTINUOUS-TIME COEFFICIENT VALUES. $\tau_{1}$ : NYSTAGMUS TIME-CONSTANT, $\tau_{2}$ : SACCADE TIME-CONSTANT, $K_{1}$ : NYSTAGMUS GAIN, $K_{2}$ : SACCADE GAIN AND T: SAMPLING INTERVAL. RIGHT: COEFFICIENT VALUES OF STATIC NONLINEARITY. $a$ : DC TERM, $b$ : LINEAR TERM, $c$ : SQUARED TERM AND $d$ CUBIC TERM.

One thousand Monte-Carlo simulations were generated in which the input-output realisation was the same but had a unique Gaussian white, zero-mean, noise sequence added to the output. Starting with the noise free (NF) case the signalto-noise ratio (SNR) of the noise sequence was decreased from $20-0 \mathrm{~dB}$ in increments of $5 \mathrm{~dB}$. The system was perturbed using a sinusoid input $(1 / 6 \mathrm{~Hz}$ frequency and 188 deg/s amplitude). A sinusoid was used as input because it is the type of input used in a typical clinical setting. The system input-output was sampled at $600 \mathrm{~Hz}$.

\section{A. Continuous-Time Parameter Estimation}

We estimated continuous-time parameters of slow and fastphase dynamics using the theoretical relationships in Table III. Since it is impossible to measure the signal at the output

\begin{tabular}{cc}
\hline CT Coefficient & DT Relationship \\
\hline$\tau_{v}=$ & $\frac{-\left(\beta_{2}, \vartheta_{2}\right) T-T}{-2+2\left(\beta_{2}, \vartheta_{2}\right)}$ \\
$G_{v} b=$ & $\frac{4\left(\beta_{3}, \vartheta_{3}\right)}{\left(\beta_{2}, \vartheta_{2}\right) T+T}$ \\
\hline \multicolumn{2}{c}{ TABLE III }
\end{tabular}

DT TO CT RELATIONSHIPS FOR PARAMETERS $\tau_{v}$, AND $G_{v} b$.

of the static nonlinearity, we consider the linear system to have unity gain and translate the overall gain onto the static nonlinearity giving an estimate as a product of the linear system and static nonlinearity gain. We deem that the best estimate of the linear system gain is the product of the linear system gain and linear coefficient of the static nonlinearity, i.e. $G_{v} b$ for $v=1$ or 2 .

The results of this study are shown in Fig. 3. Panels 3(a)

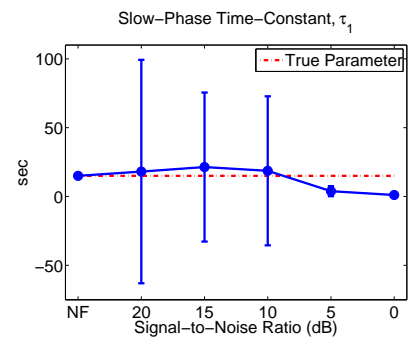

(a)

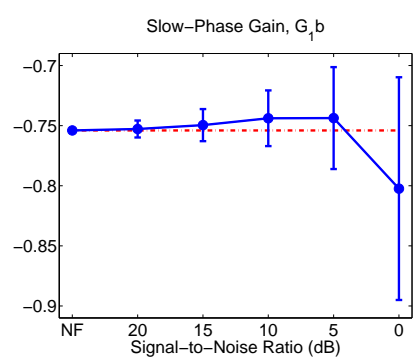

(c)

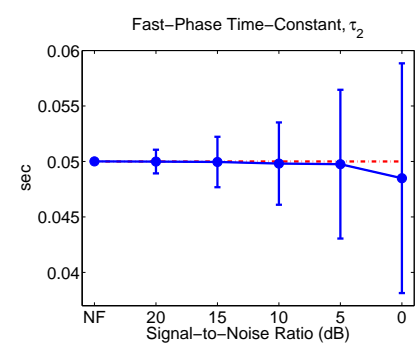

(b)

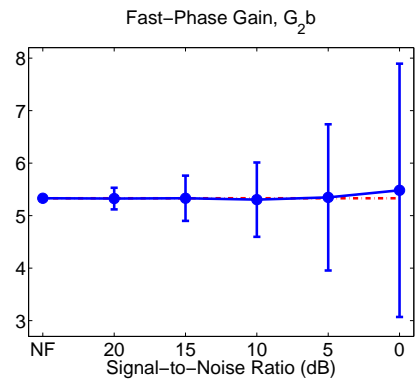

(d)
Fig. 3. Ordinate: STD about mean. Abscissa: Output SNR = NF, 20, 15, 10, $5,0 \mathrm{~dB}$, where NF denotes noise free case. (Note that the abscissa is shown in decreasing SNR which corresponds to increasing noise amplitude.).

and 3(b) display the standard deviation (STD) about the mean superimposed on the theoretically computed CT slow and fast-phase time constants. For slow-phase, the mean is close to the theoretical value and its STD includes the true value for $S N R=20-10 \mathrm{~dB}$, whilst for $S N R=5-0 \mathrm{~dB}$ the mean value is biased away from the true and the STD does not include its value. The mean of the fast-phase time constant is close to its true value and the STD includes the true value for all SNR levels. Panels 3(c) and 3(d) present the STD about the mean superimposed on the theoretically computed CT slow and fast-phase gain. The results demonstrate for all 
SNR levels the mean is close to its expected value and the STD encompasses the true value.

\section{B. Cross-Validation}

Next, we cross-validated the DT parameter estimates of VOR dynamics using a $k$-step-ahead predictor [19]. The quality of fit was assessed by computing the $\% \mathrm{QF}$ as

$$
\% Q F=\left(1-\frac{\frac{1}{N} \sum_{q=1}^{N}\left(z_{q}-\hat{z}_{q}\right)^{2}}{\frac{1}{N} \sum_{q=1}^{N}\left(z_{q}\right)^{2}}\right) \times 100
$$

where $\hat{z}$ denotes the predicted output.

Figure 4 illustrates the predicted output for a typical realisation of simulated VOR using cross-validation data (data not used for estimation). The plot shows predicted

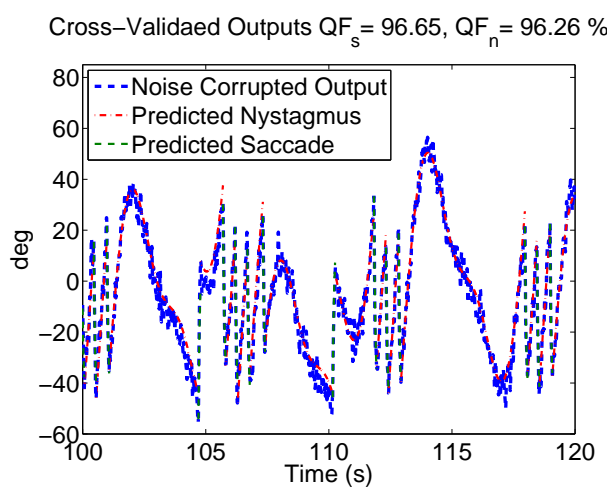

Fig. 4. Cross-validated eye position output for simulated data using a $k$-step-ahead predictor. Data noise corrupted at $15 \mathrm{~dB}$ SNR.

nystagmus (red '-.' ) and saccade (green '- -' ) superimposed on noise corrupted data (blue '- -' ) . Using our sparse matrix algorithm, the estimated parameters provide a slow and fastphase \%QF's of $96.26 \%$ and $96.65 \%$.

\section{Computation Time: Non-Sparse Versus Sparse Approach}

Lastly, we compared the computation time required by the non-sparse and sparse approach to estimate VOR parameters. For this study we computed the mean estimation time of nystagmus and saccade parameters over 1,000 trials. This was done to remove bias due to possible background process running at execution time of either approach. The analysis was performed in Matlab on a Macintosh with two $2.66 \mathrm{GHz}$ 6-Core Intel Xeon processors, $32 \mathrm{~GB} 1333 \mathrm{MHz}$ DDR3 and Mac OS X 10.6.8. This study did not take advantage of the parallel computing available on the machine since different platforms have varying numbers of cores and thus, allowing for easy scaling for a user on his/her platform. Table IV presents the result of this study. The comparison shows that

\begin{tabular}{cc}
\hline Computational Approach & Time (s) \\
\hline Non-Sparse & 202.3 \\
Sparse & 16.01 \\
\hline
\end{tabular}

TABLE IV

MEAN COMPUTATION TIME OF 1,000 MONTE CARLO SIMULATIONS FOR NON-SPARSE VERSUS SPARSE MATRIX APPROACH.

a sparse matrix approach for VOR parameter estimation is over 12 times faster than using a non-sparse approach.

\section{CONCLUSIONS}

Simulation studies demonstrate that a sparse matrix approach is a robust and efficient technique for the simultaneous analysis of nystagmus and saccade dynamics. This method offers a tool for mathematical modelling of biological processes, providing a quantitive measure to study human responses in high-g and low/zero-g environments. Moreover, this tool will have great significance for mission planners by providing them an improved ability to formulate countermeasures and, hence, increasing mission safety. The sparse technique here allows greater insight into the functionality of various reflexes by providing quantitative measures of both fast and slow ocular dynamics from a single experimental record. Consequently, this approach may be useful to estimate the coefficients of complex non-linear bifurcating systems found in biology.

\section{REFERENCES}

[1] L. Phan, I. Lee, and O. Sokolsky, "A semantic framework for multimode systems," in 17th IEEE Real-Time and Embedded Technology and Applications Symposium, vol. 17, Chicago, IL, 11-14 April 2011 2011, pp. 91-100.

[2] K. Chun and D. Robinson, "A model of quick phase generation in the vestibulo-ocular reflex," Biol. Cybern., vol. 28, pp. 209-221, 1978.

[3] H. Galiana, "A nystagmus strategy to linearize the vestibulo-ocular reflex," IEEE Trans. Biomed. Eng., vol. 38, no. 6, pp. 532-543, 1991.

[4] S. Kukreja, R. Kearney, and H. Galiana, "A least-squares parameter estimation algorithm for switched Hammerstein systems with applications to the vor," IEEE Transactions on Biomedical Engineering, vol. 52, no. 3, pp. 431-444, 2005.

[5] S. Chen and S. Billings, "Representations of non-linear systems: the NARMAX model," International Journal of Control, vol. 49, no. 3, pp. 1013-1032, 1989.

[6] C. Rey and H. Galiana, "Parametric classification of segments in ocular nystagmus," IEEE Trans. Biomed. Eng., vol. 38, no. 2, pp. 142-148, 1991.

[7] G. Golub and C. V. Loan, Matrix Computations, 3rd ed. Baltimore: The Johns Hopkins University Press, 1996.

[8] J. R. Gilbert, C. Moler, and R. Schreiber, "Sparse matrices in MATLAB: Design and implementation," SIAM Journal on Matrix Analysis and Applications, vol. 13, no. 1, pp. 333-356, 1992.

[9] J. Mellor-Crummey and J. Garvin, "Optimizing sparse matrixvector product computations using unroll and jam," International Journal of High Performance Computing Applications, vol. 18, no. 2, pp. 225236, 2004.

[10] B. Poirier, "Efficient preconditioning scheme for block partitioned matrices with structured sparsity," Numerical Linear Algebra with Applications, vol. 7, no. 7-8, pp. 715-726, 2000.

[11] Human Research Program, Integrated Research Plan, HRP-47065 Rev C ed. NASA Johnson Space Center, 2011, p. 18.

[12] J. Lackner and P. DiZio, "Space motion sickness," Exp. Brain Res., vol. 175, pp. 377-399, 2006.

[13] I. Leontaritis and S. Billings, "Input-output parametric models for nonlinear systems part I: deterministic non-linear systems," Int. J. Control, vol. 41, no. 2, pp. 303-328, 1985.

[14] R. Leigh and D. Zee, The Neurology of Eye Movements, 2nd ed. Philadelphia: F.A. Davis Company, 1991.

[15] R. Carpenter, Movements of the Eyes, 1st ed. London: Pion, 1977.

[16] R. Schmidt and F. Lardini, "On the predominance of anticompensatory eye movements in vestibular nystagmus," Biol. Cybern., vol. 23, pp. 135-148, 1976.

[17] S. Billings and W. Voon, "Least squares parameter estimation algorithms for non-linear systems," Int.J. System Sci., vol. 15, no. 6, pp. 601-615, 1984.

[18] A. George and J. W. H. Liu, Computer solution of large sparse positive definite systems, 1st ed. Englewood Cliffs, New Jersey: Prentice-Hall, 1981.

[19] L. Ljung, System Identification: Theory for the User, 2nd ed. Upper Saddle River, New Jersey: Prentice Hall, Inc., 1999. 